\title{
Philosophiques
}

\section{Habermas et la question du nationalisme : le cas du Québec}

\section{Stéphane Courtois}

Volume 27, numéro 2, automne 2000

URI : https://id.erudit.org/iderudit/004911ar

DOI : https://doi.org/10.7202/004911ar

Aller au sommaire du numéro

Éditeur(s)

Société de philosophie du Québec

ISSN

0316-2923 (imprimé)

1492-1391 (numérique)

Découvrir la revue

Citer cet article

Courtois, S. (2000). Habermas et la question du nationalisme : le cas du Québec. Philosophiques, 27(2), 377-401. https://doi.org/10.7202/004911ar

\section{Résumé de l'article}

Le but de notre article est de montrer que les écrits politiques récents de Jürgen Habermas permettent de jeter un éclairage neuf sur la situation politique du Québec. Après avoir rappelé les principaux traits de sa conception du nationalisme à la lumière de sa théorie délibérative de la démocratie, nous relevons les forces et les faiblesses de sa position. Nous montrons que ses forces consistent à rendre plausible l'idée que la reconnaissance des identités collectives n'est pas incompatible avec le système des droits individuels et que le recours à des droits collectifs n'est ni utile, ni nécessaire. Ses faiblesses, quant à elles, consistent à sous-évaluer l'État-nation comme idéal politique légitime pour les nations minoritaires et, conséquemment, dans la supposition que leur intégration à des systèmes fédératifs ouverts est l'unique voie raisonnable de solution à leurs problèmes. 


\title{
Habermas et la question du nationalisme : le cas du Québec
}

\author{
STÉPHANE COURTOIS
}

Université du Québec à Trois-Rivières

stephane_courtois@uqtr.uquebec.ca

\begin{abstract}
RÉSUMÉ. - Le but de notre article est de montrer que les écrits politiques récents de Jürgen Habermas permettent de jeter un éclairage neuf sur la situation politique du Québec. Après avoir rappelé les principaux traits de sa conception du nationalisme à la lumière de sa théorie délibérative de la démocratie, nous relevons les forces et les faiblesses de sa position. Nous montrons que ses forces consistent à rendre plausible l'idée que la reconnaissance des identités collectives n'est pas incompatible avec le système des droits individuels et que le recours à des droits collectifs n'est ni utile, ni nécessaire. Ses faiblesses, quant à elles, consistent à sous-évaluer l'État-nation comme idéal politique légitime pour les nations minoritaires et, conséquemment, dans la supposition que leur intégration à des systèmes fédératifs ouverts est l'unique voie raisonnable de solution à leurs problèmes.
\end{abstract}

ABSTRACT. - The aim of our paper is to show that Jürgen Habermas's recent political writings enable us to throw a new light on Quebec's political situation. After having remembered the central features of his conception of nationalism in the light of his deliberative theory of democracy, we call attention to its strengths and weaknesses. Its strengths are to make plausible the compatibility between collective identities and individual rights while collective rights are seen as neither useful nor necessary. Its weaknesses are to underestimate the Nation-State as a legitimate political ideal for national minorities and to consider their integration into open federal systems as the unique reasonable way out of their problems.

Que l'on puisse traiter, dans le cadre d'un article, des idées défendues par Habermas à propos du nationalisme, cela s'entend. Que l'on y ajoute «le cas du Québec ", cela paraît, du moins de prime abord, quelque peu étrange. On ne voit d'abord pas très bien ce que le concepteur de l'éthique de la discussion pourrait, au juste, avoir à dire sur les problèmes relatifs au nationalisme québécois. L'expérience allemande du nationalisme et, pourrait-on dire, l'expérience européenne en entier ont peu à voir avec l'expérience québécoise du nationalisme. Qu'est-ce qu'un philosophe aussi étranger au phénomène du multiculturalisme que peut l'être Habermas pourrait apporter de nouveau au débat ${ }^{1}$ ?

Le but de notre article est, contrairement à cette vue des choses, de montrer que les écrits politiques récents du philosophe allemand ${ }^{2}$ permettent

1. C'est là, par exemple, l'avis de Michel Seymour dans son dernier livre, La nation en question, Montréal, Éditions de l'Hexagone, 1999, p. 42 et p. 128-129.

2. Il faut compter parmi les écrits politiques récents de Habermas : Faktizität und Geltung. Beiträge zur Diskurstheorie des Rechts und des demokratischen Rechtsstaats (ciaprès : FG), Francfort, Suhrkamp, 1992 (trad. franç. par R. Rochlitz et C. Bouchindhomme, 
de jeter un éclairage neuf sur le cas québécois et le " complexe canadien » et contiennent des réflexions des plus substantielles dont les analystes et les acteurs des événements en cours au Québec et au Canada auraient tout avantage à s'inspirer - même si, comme nous le verrons, elles ne sont pas à l'abri de toute critique ${ }^{3}$. Pour le montrer, nous procéderons en trois temps.

Pour commencer, nous rappellerons les idées-forces de la conception que se fait Habermas du nationalisme à la lumière de son concept délibératif de démocratie. Trois éléments retiendront ici notre attention : tout d'abord, sa critique du nationalisme ethnique (1.1); ensuite, sa critique du modèle libéral de la citoyenneté (1.2); finalement, ses arguments en faveur d'un patriotisme constitutionnel et d'un éventuel État supranational européen (1.3). Dans un second temps, nous essaierons de tirer les conséquences de cette conception pour ce qui est du cas québécois et nous insisterons sur les forces de la position de Habermas. Nous montrerons en premier lieu qu'une telle conception, sans endosser les projets d'indépendance nationale comme celui poursuivi par le mouvement nationaliste québécois, est loin d'y être insensible, puisqu'elle fait, pour tout ordre politique fédératif, une obligation juridique de reconnaitre les identités collectives et les traditions culturelles ou nationales touchées par sa législation. Le grand intérêt du point de vue développé par Habermas est de montrer qu'une telle reconnaissance est possible sans qu'il soit pour cela nécessaire de recourir à des droits collectifs (2.1). Nous examinerons par la suite les avantages que nous semble présenter ce point de vue (2.2.). Nous terminerons notre article en insistant cette fois sur les faiblesses de la position que défend le philosophe de Francfort. Essentiellement, celles-ci consistent dans le refus, voire dans la dévaluation obstinée de l'indépendance nationale et de la constitution d'un nouvel État-nation comme voies de solution souhaitables et envisageables, au même titre que la voie fédérative, aux problèmes des nations minoritaires. Nous soutiendrons que l'État-nation doit au contraire être reconnu comme un idéal politique légitime (3.1.) et que, bien que son dépassement dans une fédération supranationale, voire dans une démocratie cosmo-

Droit et Démocratie - Entre faits et normes, Paris, Gallimard, 1997); Die Normalität einer Berliner Republik. Kleine Politische Schriften VIII (ci-après : NBR), Francfort, Suhrkamp, 1995 ; Die Einbeziehung des Anderen. Studien zur politischen Theorie (ci-après : EdA), Francfort, Suhrkamp, 1996 (trad. franç. par R. Rochlitz, L'intégration républicaine, Paris, Fayard, 1998); Die postnationale Konstellation. Politische Essays (ci-après : DpK), Francfort, Suhrkamp, 1998. Nous nous référerons dans notre article à l'édition originale allemande des oeuvres de Habermas. Les traductions françaises seront indiquées entre parenthèses, sauf lorsqu'elles ne sont pas disponibles.

3. Un seul auteur a, à notre connaissance, tenté d'appliquer la pensée politique de Habermas au cas du Québec : il s'agit de Claude Bariteau dans son article "Pour une conception civique du Québec »L'Action nationale, vol. LXXXVI, no 7, 1996, p. 105-168. Cette étude ne s'appuie toutefois que sur un seul texte de Habermas et ne manifeste aucune distance critique à son endroit. Nous nous référerons pour notre part - comme le montre la note 2 -à l'éventail le plus large possible des travaux du philosophe allemand et nous tenterons de départager, dans leur apport au débat sur le nationalisme, les éléments pertinents (section 2 de notre article) de ceux que nous estimons plus problématiques (section 3). 
politique soit incontournable dans le contexte de la mondialisation, celui-ci n'est pas opposé à, ou incompatible avec l'État-nation, mais constitue plutôt une étape supplémentaire dans l'exercice de l'autonomie politique et de la participation démocratique des citoyens (3.2).

\section{Le nationalisme selon Habermas}

\subsection{Le rejet du nationalisme ethnique}

La conception du nationalisme chez Habermas s'articule tout d'abord autour d'une réflexion sur les rapports entre nationalisme et démocratie ${ }^{4}$. Selon lui, l'idée de nation aurait deux sens, l'un plus ancien, et l'autre moderne. Dans son sens ancien, la natio doit se comprendre comme une communauté d'origine ou de provenance, prépolitique, fondée sur des traits communs ethniques ou culturels, tels que la langue, l'histoire, les moeurs et les traditions. Dans son acception moderne, qui remonterait au XVIII siècle, en particulier à partir de la Révolution française, la natio vient à se confondre avec la civitas : elle se détache de sa signification prépolitique et renvoie désormais à une communauté politiquement constituée, à une association de citoyens, source de la souveraineté politique, fondée sur des droits civiques démocratiques. Cette composante républicaine ou démocratique de la nation se serait, depuis lors, de plus en plus détachée de sa composante ethnique.

Habermas en conclut que la conscience nationale n'aura été finalement que l'instrument, le catalyseur de la genèse de l'esprit républicain, sans pour autant, cependant, qu'elle n'en forme une condition nécessaire : le lien entre nation et démocratie serait purement contingent et accidentel. Il va même plus loin : il est convaincu que la démocratie doit s'émanciper de l'Étatnation qui lui a donné naissance. Il fait peu de doute qu'il voit dans un tel affranchissement une garantie contre une rechute, qui serait désastreuse du point de vue de l'histoire européenne en général, et de l'histoire allemande en particulier, dans une forme ou une autre d'ethno-nationalisme, dont le trait essentiel est précisément de fondre ensemble ethnos et demos et d'être aveugle à la différence, centrale aux yeux du philosophe de Francfort, entre l'autonomie politique comprise comme autonomie nationale et l'autonomie politique comprise comme autonomie démocratique ou républicaine.

La première position, historiquement incarnée en Allemagne par Carl Schmitt, fonde la démocratie sur le peuple-nation : l'homogénéité nationale serait une condition nécessaire à toute démocratie et il ne saurait exister de démocratie que sous forme de démocratie nationale. L'autonomie politique

4. Pour ce qui suit : "Staatsbürgerschaft und nationale Identität » (1990), dans FG, p. 633-643 (trad. franç. dans L'intégration républicaine, p. 68-77) ; EdA, p. 130-139 et p. 157-158 (trad. franç. dans L'intégration républicaine, p. 97-105 et p. 124-125). Également : " 1989 im Schatten von 1945. Zur Normalität einer künftigen Berliner Republik », dans NBR, en particulier section III, p. 174-178. 
prend alors le sens de l'affirmation de soi collective d'un peuple-nation à l'endroit d'autres peuples-nations. Contre cette vue des choses, Habermas s'est efforcé, dans les travaux qu'il a entrepris au cours des dernières années sur le droit et la démocratie, de proposer une conception procédurale du principe de la souveraineté populaire ${ }^{5}$. Celle-ci renonce à une lecture trop concrétiste de ce principe, comme chez Schmitt, en faveur d'une conception plus abstraite, ancrant le principe de la souveraineté populaire dans une procédure formelle de la formation politique de la volonté et de l'opinion. Cette procédure, appelée "procédure démocratique " (demokratisches Verfahren), est chargée, pour tous ceux qui désirent régler légitimement leur vie commune au moyen du droit, de fonder la présomption de la rationalité des résultats d'une pratique délibérative publique. Le sujet de l'autodétermination démocratique n'est plus hypostasié dans un peuple-nation culturellement homogène, il se mue plutôt en une association anonyme de sujets de droit libres et égaux, se rapportant les uns aux autres par des échanges communicationnels selon différents modèles de discussion publique, organisés non seulement à l'intérieur (dans les arènes parlementaires) mais également à l'extérieur (dans les espaces publics informels) des corps politiques et ce, sans égard à leur provenance ethnique ou culturelle. L'autonomie politique a ici non plus le sens collectiviste et exclusif d'une affirmation de la souveraineté nationale vis-à-vis d'autres États-nations, mais celui, inclusif, d'une pratique d'autolégislation et d'autodétermination des citoyens à l'intérieur d'une communauté politique aux frontières multiples, non d'emblée délimitée par des frontières ethniques ou nationales (communauté pouvant s'incarner, comme nous le verrons dans les sections suivantes, non seulement dans l'État-nation classique, mais également dans des fédérations supranationales, voire dans des institutions non étatiques mondiales).

\subsection{La critique du modèle libéral de la citoyenneté}

Pour être en mesure de saisir correctement le statut qu'occupent les revendications nationalistes dans la pensée de Habermas, il importe d'aller au-delà de sa réflexion sur les rapports entre nationalisme et démocratie, et de s'attarder quelque peu sur l'analyse qu'il propose des rapports entre État de droit et démocratie, entre le système des droits individuels et la citoyenneté démocratique. Bien que Habermas rejette sans détour le nationalisme ethnique, il n'endosse pas pour autant le modèle libéral de la citoyenneté. Si l'erreur du nationalisme ethnique est de fondre le républicanisme dans le nationalisme, la citoyenneté démocratique dans l'appartenance nationale ou ethnique, l'identité politique dans l'identité culturelle, l'erreur de la doctrine libérale est de ne

5. Voir FG, en particulier p. 112-135 et p. 367-382 (trad. franç. dans Droit et démocratie, p. 100-120 et p. 327-340). Également : «Volkssouveränität als Verfahren » (1988), dans FG, p. 600-631. 
voir dans le premier des deux termes que l'instrument de la réalisation des libertés individuelles. Elle fait certes valoir, comme Habermas, l'importance d'une démarcation conceptuelle, voire l'indépendance logique entre natio et civitas, entre ethnos et demos ; cependant, selon le philosophe de Francfort, la manière libérale de conceptualiser la civitas serait si étroite qu'elle ne laisserait plus aucune place à la natio, et conduirait au refus de reconnaître la spécificité des identités culturelles et nationales. Examinons plus avant les principaux traits de sa critique du modèle libéral de la citoyenneté ${ }^{6}$.

Habermas s'accorde avec plusieurs des critiques que les communautariens ont adressées jusqu'ici à la doctrine libérale, sans pour autant en partager les solutions ${ }^{7}$. Il s'accorde notamment avec la critique faite par Charles Taylor ${ }^{8}$ de la vision strictement instrumentale qu'aurait le libéralisme de l'autonomie publique et des libertés politiques, où le citoyen ne s'associe avec les autres citoyens qu'en vue d'influencer le pouvoir politique dans le sens de ses intérêts privés. À la différence des communautariens cependant, Habermas ne milite aucunement en faveur du rétablissement dans les moeurs publiques d'une forme d'esprit civique national. Le sens de sa critique est plutôt le suivant : le libéralisme méconnaîtrait le caractère co-originaire de l'autonomie privée et de l'autonomie publique'. S'il n'existe aucun lien conceptuel entre nationalisme et démocratie et si le nationalisme ethnique commet l'erreur de fusionner maladroitemnent les deux, il existe par contre, prétend

6. Pour ce qui suit, voir surtout: "Kampf um Anerkennung im demokratischen Rechtsstaat»(1992), p. 237-276 et "Drei normative Modelle der Demokratie » (1992), en particulier p. 277 sq., tous deux dans EdA (trad. franç. dans L'intégration républicaine, p. 205243 et p. 260 sq.). Pour une critique du modèle libéral de la citoyenneté en relation au problème de la mondialisation, voir " Die postnationale Konstellation und die Zukunft der Demokratie ", dans $D p K$, p. 140-144.

7. Habermas rejoint par exemple les communautariens dans leur rejet de la conception atomiste de l'individu, à laquelle il oppose une conception intersubjectiviste de la personne qui remonte à ses travaux, développés au cours des années 70, sur l'ontogénèse et la théorie de la socialisation. Pour une vision d'ensemble des relations entre le modèle de la démocratie délibérative défendu par Habermas et les conceptions libérale et communautarienne, voir « Drei normative Modelle der Demokratie », dans EdA, p. 277-305 (trad. fr. dans L'intégration républicaine, p. 259-274) ; FG, surtout p. 324-348 et p. 358-366 (trad. fr. Droit et démocratie, p. 290-310 et p. 320-327). Pour une discussion d'ensemble des trois conceptions, voir Brumlik, M. et Brunkhorst, H., dir., Gemeinschaft und Gerechtigkeit, Francfort, Fischer Taschenbuch Verlag, 1993 (2e édition 1995).

8. Taylor, Charles, "Cross-Purposes : The Liberal-Communitarian Debate », dans Rosenblum, Nancy L., dir., Liberalism and the Moral Life, Cambridge, Mass., Harvard University Press, 1989, voir p. 178 sq.

9. Sur les rapports entre autonomie publique et privée et sur leur caractère cooriginaire, voir tout le chapitre III de $F G$; voir également $E d A$, p. 163-164, p. 242 sq. et p. 298 sq. (trad. franç. p. 130, p. 210 sq. et p. 280 sq.). Mentionnons également que la question des relations entre autonomie privée et publique forme l'un des points de désaccord décisifs entre Habermas et Rawls dans leur controverse récente. Voir Rawls, John et Habermas, Jürgen, Débat sur la justice politique, trad. par R. Rochlitz, Paris, Les Éditions du Cerf, 1997, en particulier p. 40 sq. et p. 83 sq. 
Habermas, un lien interne, conceptuellement nécessaire, entre l'État de droit (et le système des droits individuels dont il se porte garant) et la démocratie : c'est le libéralisme qui aurait tort, cette fois, de ne pas le reconnaître.

Si l'État de droit doit promouvoir et protéger les droits individuels, il revient aux citoyens de déterminer eux-mêmes, étant les destinaires de ces droits, les conditions significatives de leur exercice. Et ceci ne peut se faire, selon Habermas, qu'au moyen de délibérations menées dans l'espace public politique au sujet de l'interprétation de leurs besoins, et donc par un usage communicationnel ou intersubjectif (non instrumental) de l'autonomie publique et des libertés politiques dont ils disposent. De telles délibérations attirent l'attention sur les contextes de vie particuliers dans lesquels a à s'enraciner le système des droits individuels. Comme l'a montré Habermas à plusieurs reprises, c'est précisément cette sensibilité au contexte, cette "imprégnation éthique » (ethische Imprägnierung) des normes du droit, lesquelles sont à la fois l'expression du contenu universel des droits fondamentaux et le reflet particulier d'une forme de vie, qui les distingue des normes morales ${ }^{10}$. Alors que ces dernières doivent se faire les véhicules - du moins selon les approches déontologiques de la théorie morale - des notions de justice et d'impartialité indépendantes de toutes les conceptions de la "vie bonne " privilégiées par les membres de la société, il n'en va pas de même des premières, qui ne se situent pas au même niveau d'abstraction : elles ont aussi à réguler le contexte de vie des membres d'une communauté culturelle, politique, nationale concrète et, pour cette raison, leur validité est contextuelle. Pour un législateur, les normes juridiques sont légitimes, non pas seulement lorsqu'elles peuvent être justifiées sous un aspect déontologique, à partir de raisons morales, mais également lorsqu'elles permettent aux membres d'une communauté politique, culturelle ou nationale donnée de parvenir à un accord éthico-politique sur les conceptions du bien et les valeurs préférentielles à réaliser par eux.

Habermas opère ici, ni plus ni moins, une correction " républicaine » du modèle libéral de la citoyenneté en montrant que la citoyenneté démocratique ne peut se ramener à la simple jouissance de l'autonomie privée, que celle-ci ne peut pleinement se réaliser que si l'exercice de l'autonomie publique, entendue au sens d'une pratique délibérative des citoyens, est promue de façon active. Il montre que l'on ne peut rejeter du revers de la main les revendications identitaires émanant d'une culture ou d'une nation sous couvert d'une protection des droits individuels puisque c'est toujours dans le contexte d'une tradition culturelle ou d'une nation particulière que doit être interprété, par les citoyens, le contenu universaliste des droits individuels. Nous tirerons plus loin les implications de cette prise en considération des contextes pour ce qui est de la situation particulière du Québec. 


\subsection{Patriotisme constitutionnel et État supranational}

On ne peut avoir une vue d'ensemble satisfaisante de la conception que se fait Habermas du nationalisme si on omet d'examiner le sens de son «patriotisme constitutionnel » et de ses idées en faveur d'un futur État fédéral européen. Son rejet de la doctrine libérale n'est qu'un rejet partiel : il n'est dirigé, rappelons-le, que contre sa vision trop étroite du rôle des libertés politiques dans le contexte d'une démocratie et son inaptitude à prendre en compte les milieux de vie particuliers, culturels ou nationaux, dans lesquels ont à s'implanter les droits individuels. Il laisse intact son rejet du nationalisme ethnique. Il ne touche pas non plus à la valeur universaliste des droits individuels, lesquels, au contraire, sont précisément l'objet du " patriotisme constitutionnel » dont se réclame Habermas, que nous allons examiner ici.

\subsubsection{Le patriotisme constitutionnel est-il une forme de nationalisme civique?}

Le patriotisme constitutionnel, tel que le conçoit Habermas, exige des citoyens l'identification à des principes juridiques ou constitutionnels abstraits, dont la visée est universelle - principes de justice, de démocratie, de citoyenneté, droits de la personne, etc. - qui sont pour la plupart inscrits dans le texte fondamental des États de droit démocratiques modernes. Son but est de forger une citoyenneté démocratique essentiellement fondée sur le droit ${ }^{11}$. Il est clair ici que ce type de patriotisme doit être mis à distance d'un patriotisme de type national ou ethnique, c'est-à-dire de toute identification à une communauté nationale fondée sur certaines valeurs communes (au sens de C. Taylor). Ce qui est moins clair, c'est de savoir si le patriotisme constitutionnel doit être identifié au nationalisme civique. Nous soutiendrons que non : il se présente en effet comme une alternative au nationalisme et non comme une forme de nationalisme. Le nationalisme civique semble présumer, si l'on en juge à sa dénomination même, que les principes juridiques et constitutionnels fondant la citoyenneté démocratique doivent s'ancrer dans un "État territorial » (non nécessairement homogène au plan ethnique) et ont pour mission de forger une identité "nationale ». Le patriotisme constitutionnel se distingue à cet égard du nationalisme civique de deux manières. Tout d'abord, il n'a pas nécessairement d'ancrage national, ni de frontières nationales. Il repose en effet sur des principes moraux et de justice dont la visée est universelle et qui ne peuvent être limités au texte constitutionnel de tel ou tel État particulier. En second lieu, et en conséquence de ce qui précède, le patriotisme constitutionnel est l'expression, non d'une conscience nationale, mais bien plutôt d'un cosmopolitisme radical qui ne peut fonder qu'une identité politique abstraite, jamais une identité

11. Voir «Staatsbürgerschaft und nationale Identität » dans FG, p. 649 et EdA, p. 189 (trad. franç. dans L'intégration républicaine, p. 83 et p. 155). 
«nationale $»^{12}$. En d'autres termes, bien qu'il ne soit pas incompatible avec les structures de l'État-nation territorial, le patriotisme constitutionnel ne peut à lui seul suffire à fonder une identité nationale. Pour fonder une identité nationale, il faut, en plus du patriotisme constitutionnel, une culture nationale, c'est-à-dire certaines traditions ou histoires nationales particulières à la lumière desquelles seront interprétés les principes universalistes du patriotisme constitutionnel ${ }^{13}$. Si la culture nationale est nécessaire pour fonder une identité nationale, elle n'est, en revanche, aucunement nécessaire pour fonder le patriotisme constitutionnel. Habermas insiste fréquemment sur le fait que, pour pouvoir prendre racine et se connecter aux motivations et aux mentalités des citoyens, le patriotisme constitutionnel doit uniquement pouvoir compter sur une certaine culture politique de type libéral ou civique, laquelle ne doit en aucun cas être confondue avec la culture nationale d'un peuple quelconque, majoritaire ou minoritaire sur un territoire donné : selon Habermas, la culture politique nécessaire au patriotisme constitutionnel, en tant que culture minimale de convergence, peut et doit être partagée à un niveau supranational, sans devoir s'appuyer sur une quelconque provenance commune, ethnique ou culturelle des citoyens ${ }^{14}$.

\subsubsection{Vers un dépassement de l'État-nation}

Habermas croit que le patriotisme constitutionnel, en tant qu'expression d'une identité politique postnationale, serait le chemin sur lequel seraient actuellement engagés, à tout le moins dans l'Europe de Maastricht, la plupart des États-nations classiques. En ce sens, l'État-nation serait pour lui chose du passé et les mouvements nationalistes auraient aujourd'hui à relever le défi de redéfinir leurs projets politiques dans le sens de stratégies d'intégration à des unités supranationales plutôt que dans le sens d'une sécession. Une telle stratégie serait entre autres rendue nécessaire par la mondialisation des marchés et la perte d'influence des États-nations sur les grandes orientations de l'économie à l'échelle internationale. Les deux piliers incontournables de l'État-nation que sont la souveraineté extérieure et la souveraineté intérieure seraient aujourd'hui, selon Habermas, remis en question et leur

12. Sur les ambiguités mêmes liées à la notion de "nationalisme civique ", voir le texte des plus intéressants de Wayne Norman, "Le paradoxe du nationalisme civique », dans Laforest, Guy et de Lara, Philippe, dir., Charles Taylor et l'interprétation de l'identité moderne, Les Presses de l'Université Laval, 1998, p. 155-170.

13. Habermas aime à cet égard donner l'exemple de la Suisse. Voir «Staatsbürgerschaft und nationale Identität », dans FG, p. 642 et p. 651 (trad. franç. dans L'intégration républicaine, p. 77 et p. 85 ).

14. Sur les différences entre culture "politique» et culture «nationale» et sur les relations entre les deux, voir, entre autres, "Staatsbürgerschaft und nationale Identität ", dans $F G$, p. 642 , p. 651 et p. 659 et EdA, p. 142-143 et p. 190 (trad. franç. dans L'intégration républicaine, p. 77 , p. 85, p. 93, p. 108-109 et p. 156). 
distinction rendue de plus en plus floue ${ }^{15}$. Au plan de la souveraineté extérieure, l'idée classique d'une politique de la puissance, d'une affirmation des nations dans l'arène internationale et d'une stratégie d'intimidation réciproque au moyen des forces armées serait de plus en plus remplacée par l'idée d'une coopération et d'un partage, au plan supranational, des risques planétaire encourus, par l'idée d'un pouvoir exercé " en douceur » (le "soft power ») et de stratégies d'intervention non violentes (sanctions économiques, interventions humanitaires, etc.). Au plan de la souveraineté interne, non seulement la dénationalisation de l'économie et la mondialisation des marchés entraîneraient-elles la perte de contrôle, par l'État-nation, de sa politique économique ou monétaire interne et des conditions de production en général, mais la diffusion planétaire d'une culture de masse (en bonne partie dominée par les États-Unis), où les mêmes styles de consommation, les mêmes films, les mêmes modes musicales ou vestimentaires se répandent dans tous les pays et uniformisent les styles de vie, voire l'imposition de l'anglais comme langue officielle des affaires et comme mode de communication international, rendraient de plus en plus précaires les conditions d'une véritable souveraineté culturelle elle-même ${ }^{16}$.

La solution au défi de la mondialisation ne peut consister selon Habermas que dans la recherche de moyens d'action politique au plan supranational, dans la formation d'une citoyenneté démocratique et d'un réseau de communication entre citoyens qui franchissent les frontières nationales, autrement dit, dans la constitution d'un espace public transnational s'appuyant sur une culture politique libérale commune et porté par des mouvements sociaux (c'est-à-dire par des groupes d'intérêts, des organisations de citoyens, des mouvements civiques, etc.) politiquement mobilisés au sein de la société civile. Ses réflexions se sont jusqu'ici arrêtées sur deux stratégies : (1) la formation d'un État fédéral européen doté d'une constitution démocratique propre (objet d'un patriotisme constitutionnel), qui serait formé de plusieurs nationalités et où l'on parlerait plusieurs langues, l'anglais demeurant toutefois la langue principale de communication. Ce que Habermas a en vue, c'est un véritable parlement européen pourvu de compétences accrues et ce, non pas d'abord au plan économique (domaine auquel le confine l'entente de Maastricht), mais au plan politique, avec un système de partis conçu pour des arènes européennes ${ }^{17}$; (2) la participation démocratique de

15. Voir « Kants Idee des ewigen Friedens - aus dem historischen Abstand von 200 Jahren »(1995), dans EdA, p. 203 (trad. franç. dans L'intégration républicaine, p. 172) ; «Die postnationale Konstellation und die Zukunft der Demokratie ", dans DpK, p. 109.

16. Voir «Die postnationale Konstellation », dans $D p K$, p. 114-115.

17. Habermas a développé ses réflexions sur un éventuel État fédéral européen dans les textes suivants. (1) Dans FG : la section II de « Staatsbürgerschaft und nationale Identität », p. 643-651. (2) Dans EdA : les sections V et VI de " Der europäische Nationalstaat - Zu Vergangenheit und Zukunft von Souveränität und Staatsbürgerschaft» (1996), p. 145-153 ; la section V de "Inklusion - Einbeziehen oder Einschließen ? ", p. 180-184 et finalement, "Braucht 
citoyens ou d'organisations non étatiques (on pense ici à Greenpeace ou à Amnesty International) à un espace public planétaire encore en formation (les sommets mondiaux, sous l'égide de l'ONU, sur l'écologie, la démographie, la pauvreté, etc., en seraient des exemples) rendant possible la création d'une démocratie cosmopolitique et la mise en œuvre d'une politique interne mondiale (sans État mondial, comme chez Kant) ${ }^{18}$.

Quelles leçons pouvons-nous et devons-nous tirer de ces réflexions pour ce qui est de notre situation au Québec, notamment dans ses relations avec le reste du Canada ? C'est ce qu'il nous faut examiner à présent.

\section{Le cas du Québec}

Comme les analyses qui précèdent le montrent clairement, les projets politiques de type nationaliste, qu'il soit question de la préservation des prérogatives des États-nations existants ou de la constitution de nouveaux Étatsnations par voie de sécession, sont dépassés aux yeux de Habermas : l'Étatnation n'est plus en mesure de réunir les moyens d'action politique nécessaires pour contrebalancer les effets de la mondialisation et l'issue est à chercher, entre autres, du côté des regroupements fédératifs ${ }^{19}$. S'ensuit-il que Habermas devrait d'emblée être favorable à une fédération comme le Canada ? À première vue et en principe, cela semble plus que plausible. Mais

Europa eine Verfassung ? Eine Bemerkung zu Dieter Grimm » (1995), p. 185-191. Tout ce qui précède est traduit dans L'intégration républicaine, p. 78-85, p. 111-119, p. 146-150 et p. 151157. (3) Dans $D p K$ : « Aus Katastrophen lernen ? Ein zeitdiagnostischer Rückblick auf das kurze 20. Jahrhundert ", en particulier la section III et p. 84-85 et la section IV à " Die postnationale Konstellation », p. 135-156. (4) Dans «The European Nation-State and the Pressures of Globalization », New Left Review, vol. 235, 1999, p. 46-59, voir en particulier p. 55-59.

18. Habermas a ici développé ses réflexions dans les textes suivants. (1) Dans $E d A$ : "Kants Idee des ewigen Friedens », p. 218-220 (trad. franç. dans L'intégration républicaine, p. 187-189). (2) Dans $D p K$ : la section IV à «Aus Katastrophen lernen ? ", p. 84-90 et la section V à " Die postnationale Konstellation ", p. 156-169. Pour une vision d'ensemble du cosmopolitisme de Habermas par rapport à celui de Kant, voir McCarthy, Thomas, "On Reconciling Cosmopolitan Unity and National Diversity ", Public Culture, vol. 11, no 1, 1999, p. 175-208.

19. Le caractère dysfonctionnel de l'État-nation dans le contexte de la mondialisation n'est que l'un des arguments avancés par Habermas contre le nationalisme. D'autres arguments sont également à considérer : (1) la création d'un État souverain n’éliminerait pas le problème des minorités mais ne ferait que générer de nouvelles minorités nationales ; (2) les frontières d'un État souverain ne seraient aucunement garanties en vertu du droit international à l'autodétermination des peuples, puisqu'il est impossible de déterminer juridiquement comment doit se composer la totalité fondamentale des personnes qui se rassemblent pour fonder un État. Pour ces arguments, voir EdA, en particulier p. 139-142 (trad. franç. p. 106-108), p. 167-171 (trad. franç. 133-137) et p. 256 (trad. franç. p. 223). Habermas rejoint ici les arguments antisécessionnistes d'Allen Buchanan. Voir à ce sujet : Buchanan, Allen, "The Morality of Secession ", dans Kymlicka, Will, dir., The Rights of Minority Cultures, Oxford, Oxford University Press, 1995, p. 350-374. Bien que les arguments précédents méritent l'attention, nous nous concentrerons, dans la section 3 , sur celui de la mondialisation uniquement, qui nous apparaît le plus important à être formulé par Habermas contre le nationalisme. 
une analyse plus nuancée risque fort de décevoir les fédéralistes canadiens partisans du statu quo politique et constitutionnel qui, bien que minoritaires au Québec, restent majoritaires dans le reste du Canada.

Tout d'abord, le fameux « patriotisme constitutionnel » dont se réclame Habermas ne peut servir à légitimer quelque chose comme le fédéralisme canadien, du moins dans son état actuel : il se présente, rappelons-le, non comme une forme de nationalisme, mais comme une alternative au nationalisme. Certes, il pourrait convenir à un Canada multinational à l'intérieur duquel les principes juridiques et constitutionnels fondant un tel patriotisme seraient clairement érigés à un niveau supranational et pourraient être interprétés à la lumière de chacune des histoires et traditions nationales particulières composant le Canada. Mais il ne peut convenir au Canada actuel, un Canada se percevant comme une "nation " bilingue et multiculturelle : nous l'avons vu, le patriotisme constitutionnel ne peut à lui seul fonder une identité nationale, une culture politique libérale reposant sur des principes moraux ou de justice universalistes ne pouvant à elle seule fonder une culture nationale, encore moins se confondre avec elle ${ }^{20}$. Les fédéralistes se retrouvent finalement devant un dilemme : ou bien fonder l'État canadien sur un patriotisme constitutionnel et renoncer par le fait même à se percevoir comme une nation, ce qui implique de reconnaître le caractère multinational du Canada ; ou bien continuer de se percevoir comme une nation et chercher ailleurs que dans un patriotisme constitutionnel ses traits identitaires, puisqu'il ne peut à lui seul fonder une identité et une culture nationales typiquement canadiennes.

En outre, et bien que Habermas prenne ses distances à l'endroit des projets politiques nationalistes, des éléments centraux de sa réflexion sur le nationalisme font valoir, nous l'avons vu, la nécessité d'une prise en considération des revendications identitaires nationales et s'opposent à une politique «neutre » à l'endroit des cultures et nations minoritaires, insensible aux différences qu'elles présentent. C'est pourquoi les prétentions autonomistes des multiples mouvements nationalistes à travers le monde, comme celui du Québec, exigent d'être reconnues, à défaut de quoi la quête d'indépendance nationale conserve toute sa raison d'être. C'est ce qui ressort de la discussion de Habermas avec Charles Taylor sur la politique de la reconnaissance ${ }^{21}$. Avant de nous pencher sur les points faibles de sa position sur le nationalisme, nous allons au préalable exploiter ses points forts, en relation avec cette discussion, qui consistent à montrer que la reconnaissance des particularités nationales comme celle du Québec est tout à fait compati-

20. Sur ce point, nous rejoignons tout à fait, répétons-le, les analyses de Wayne Norman (voir note 12).

21. Pour la position précise de Habermas sur la situation du Québec, voir «Kampf um Anerkennung ", dans EdA, p. 248-249 et p. 256 (trad. franç. dans L'intégration républicaine, p. 216 et p. 223). La rédaction de l'essai date du début de 1993. Il succède donc à l'accord du Lac Meech qui venait d'échouer et précède de peu celui de Charlottetown. Il ne tient donc pas compte de l'échec de ce dernier ni des résultats du référendum de 1995. 
ble avec le système des droits individuels (2.1.). Nous soutiendrons par la suite que la position de Habermas, qui est celle d'une politique de la reconnaissance qui refuse de s'appuyer sur l'idée de droits collectifs, doit être préférée à celle de Taylor pour plusieurs raisons (2.2.).

\subsection{La reconnaissance du Québec est-elle compatible avec la doctrine individualiste des droits?}

On sait que Taylor défend, dans sa longue contribution à l'ouvrage Multiculturalisme. Différence et démocratie ${ }^{22}$, la thèse de la nécessité de droits collectifs - bien que l'expression n'apparaisse nulle part dans son texte ${ }^{23}-$ c'est-à-dire de garanties constitutionnelles explicites à propos de la survie des cultures menacées. Il pose la question centrale de savoir si la doctrine individualiste des droits de nature libérale permet de reconnaître les identités collectives et les formes de vie socioculturelles menacées, comme au Québec. Il répond par la négative. Selon lui, l'universalisation du droit à des libertés individuelles égales, c'est-à-dire à des chances égales pour chacun de poursuivre sa conception particulière du bien, conduirait au nivellement de toutes les différences, sociales ou culturelles. En d'autres termes, les droits subjectifs libéraux entreraient en concurrence avec le principe du respect des différences culturelles et feraient obstacle à la poursuite de desseins collectifs en matière de langue et de culture, comme au Québec. C'est pour corriger cette situation que Taylor suggère une alternative au modèle libéral, un contremodèle " communautarien » qui, certes, continuerait à garantir comme le premier les droits individuels fondamentaux, mais dans lequel l'État pourrait dans certains cas intervenir et imposer des limites à de tels droits par des mesures légales ou constitutionnelles destinées à garantir la survie collective de certaines cultures ou nations menacées. C'est donc, par exemple, essentiellement sur la base du droit collectif à la survie culturelle du peuple québécois que l'on pourrait justifier les multiples restrictions qu'impose la Loi 101 aux droits des francophones, des anglophones ou des nouveaux arrivants d'éduquer ou non leurs enfants dans une école anglaise.

Or Habermas ne croit pas qu'il soit nécessaire, pour la prise en compte des particularités culturelles ou nationales, d'en arriver à une telle remise en question de la structure individualiste du système des droits. Il suffit selon lui de réaliser ce système de manière conséquente, ce que ne ferait pas, ou ce que

22. Taylor, Charles, Multiculturalisme. Différence et démocratie, trad. de l'américain par Canal, D.-A, Paris, Aubier, 1994. (Ouvrage original : Taylor, C. \& Gutman, A., Multiculturalism and "The Politics of Recognition ", Princeton, Princeton University Press, 1992).

23. Taylor lui-même se demande, à la fin de son essai, s'il fait sens d'exiger comme un « droit» le respect des différences culturelles, craignant que cela ne conduise à une forme de multiculturalisme homogénéisant (voir Multiculturalisme, p. 93 sq.). C'est toutefois bel et bien l'idée d'un droit collectif à la survie culturelle qui ressort, sinon dans la lettre, du moins dans l'esprit de son étude, en particulier lorsqu'il traite du cas du Québec dans la section IV. 
ne ferait que de manière partielle et restrictive, la doctrine libérale. Il s'accorde donc avec Taylor sur les objectifs, à savoir sur la nécessité d'une politique de la reconnaissance des identités culturelles et nationales menacées ; il s'en dissocie au niveau des moyens : s'il s'agit bel et bien de corriger l'étroitesse de vues de la lecture libérale du système des droits, il est inutile pour cela de remettre en question le noyau individualiste de ce système en faveur de droits collectifs $^{24}$. Que la prise en compte des contextes particuliers dans lesquels ont à s'exercer les droits individuels, que le respect de l'intégrité des formes de vie socioculturelles découlent d'une réalisation conséquente du système des droits fondamentaux, c'est ce que révèlent trois thèses "fortes » propres à la théorie du droit et de la démocratie de Habermas, dont certaines ont été mentionnées dans les sections précédentes ${ }^{25}$ :

1) Appelons la première thèse "intersubjectiviste ": les personnes ne deviennent des sujets de droit autonomes que grâce à leur socialisation à l'intérieur de contextes de vie intersubjectivement partagés, que l'on peut concevoir comme des réseaux d'interactions communicationnelles. C'est dans ces contextes et dans ces réseaux que se forge leur identité individuelle, laquelle ne peut être séparée des identités collectives puisqu'elle ne se stabilise que par l'acquisition de biens culturels communs, comme la langue maternelle par exemple. L'intégrité même des sujets de droit ne peut donc être garantie sans le respect de ces contextes formateurs de l'identité. C'est ce que dit Habermas : "Les personnes en général, y compris les sujets de droit, ne deviennent des individus que grâce à la socialisation. Sous cette

24. Mentionnons ici que l'utilisation faite par Habermas de la notion de «droits collectifs " demeure imprécise (ce qu'elle est de toute manière). Habermas n'est pas opposé à ce que l'on pourrait appelé des droits collectifs partiels ou limités, entendus par exemple au sens des mécanismes d'accomodements des minorités prévus par Will Kymlicka (dans son ouvrage Multicultural Citizenship : A Liberal Theory of Minority Rights, Oxford, Oxford University Press, 1995, en particulier aux chapitres 6 et 7). Les droits collectifs sont pour lui acceptables à deux conditions : (1) Ils doivent rester subordonnés au système des droits individuels. Une mesure législative visant une collectivité déterminée ne peut limiter des droits individuels qu'à la condition de rétablir une inégalité historique ayant jusqu'ici empêché les membres d'un groupe économique, culturel, sexuel ou racial donné de jouir pleinement de l'égalité juridique. On a en ce cas affaire à des droits collectifs " partiels » ou "limités » en ce qu'ils ne sont que des moyens pour corriger une distribution inéquitable des droits individuels, et non des droits qui entendent les supplanter. (2) Ces droits doivent de plus rester à la disposition des principaux intéressés, et donc favoriser leur autonomie et non pas s'opposer à leur autonomie, chose qui pourrait survenir si on avait affaire, non pas à de simples mesures législatives (lesquelles peuvent être abrogées assez facilement), mais à des garanties constitutionnelles (concernant la langue, la culture, etc.) qui peuvent, à long terme, étant beaucoup moins flexibles, en venir à s'opposer à la volonté des membres du groupe. Bref, Habermas s'oppose à des droits collectifs qui auraient une portée équivalente aux droits individuels et qui leur feraient concurrence. Pour une analyse nuancée des difficultés et des ambiguïtés liées à la notion de droit collectif, voir Hartney, M., "Some Confusions Concerning Collective Rights ", dans Kymlicka, The Rights of Minority Cultures, p. 202-320. Voir également Kymlicka, Multicultural Citizenship, p. 45-48.

25. Voir section 1.2. 
prémisse, une théorie des droits bien comprise requiert précisément une politique de la reconnaissance qui protège l'intégrité de l'individu, y compris dans les contextes de vie qui forment son identité ${ }^{26}$. »

Dans cette perspective, les diverses dispositions prises par l'État québécois pour se protéger contre la culture majoritaire anglo-saxonne (les multiples réglementations que contient la Loi 101) peuvent se comprendre, à la lumière de la thèse intersubjectiviste, non pas, comme le pense Taylor, comme l'expression d'un droit collectif à la survie culturelle, mais plutôt comme un moyen législatif visant à assurer la prépondérance des droits individuels linguistiques ou culturels des francophones face à d'autres droits individuels. Cette prépondérance est justifiée par la nécessité, pour les membres d'une importante minorité native de langue française résolus à perpétuer leur culture dans un contexte nord-américain essentiellement anglophone, de protéger leurs milieux de socialisation. Certes, les droits individuels des anglophones et des immigrants vivant au Québec, ou des francophones eux-mêmes voulant éduquer leurs enfants dans des écoles anglaises, sont limités. Mais, à la lumière de la thèse intersubjectiviste, ils le sont, non par des droits collectifs, mais par d'autres droits individuels jugés, en regard du contexte, déterminants. L'existence d'une forme de vie collective qui serait propre à la société québécoise n'est ici nullement contestée ; seule l'est la nécessité de recourir à des droits collectifs pour la reconnaître et la protéger. Selon la thèse intersubjectiviste de Habermas, la pleine reconnaissance de l'intégrité des sujets de droit individuels implique la reconnaissance de leur contexte collectif de socialisation. Bien entendu, cette reconnaissance doit demeurer à leur disposition. Seuls les individus concernés sont en mesure de déterminer si leur milieu de socialisation doit être protégé en priorité, ou, à l'inverse, s'ils désirent renoncer à leur héritage culturel : voilà des questions qui restent soumises à l'exercice de leur liberté démocratique, au moyen de laquelle seulement les intéressés sont en mesure de faire valoir leurs besoins véritables et de s'assurer d'une prise en compte adéquate de leur situation.

(2) La sensibilité du système des droits fondamentaux aux contextes socioculturels est également au coeur d'une seconde thèse, examinée préalablement, et qui porte sur la nature même des normes du droit, celle de l' "imprégnation éthique » des ordres juridiques. Selon cette thèse, les systèmes juridiques sont à la fois l'expression du contenu universel des droits fondamentaux et le reflet particulier d'une forme de vie, ce qui les distingue des normes morales. L'erreur de la lecture libérale du système des droits serait ici de ne pas voir que la « neutralité » de l'ordre juridique doit consister, non pas à faire abstraction des contextes dans lesquels il a à s'implanter et à niveller toutes les différences que présentent les milieux socioculturels, mais uniquement à éviter de promouvoir de manière unilatérale et discriminatoire une seule conception de la vie bonne au détriment de toutes les autres.

26. "Kampf um Anerkennung ", p. 243 (trad. franç. p. 210-211). Voir également p. 258 (trad. franç. p. 225). Habermas résume sa position dans NBR, p. 84. 
Un État canadien multinational serait à n'en pas douter à même de rencontrer les obligations découlant de cette "imprégnation éthique » des systèmes juridiques, en reconnaissant les spécificités et les différences, historiquement significatives, que présentent le Québec et les nations autochtones. Reconnaître ces particularités et ces différences, ce serait reconnaître que ce n'est que dans les contextes de ces traditions culturelles ou nationales spécifiques que peut être interprété et acquérir une réelle signification le système des droits individuels (ou les principes civiques d'un patriotisme constitutionnel). Le Canada actuel, bilingue et multiculturel, est très éloigné d'une telle reconnaissance : en se dotant d'une Constitution sans le consentement du Québec, il a commis l'erreur de penser qu'on ne peut consentir de manière non discriminatoire les mêmes droits fondamentaux à tous les Canadiens qu'en aplanissant les différences nationales ou culturelles, qu'en mettant toutes les cultures sur un même pied d'égalité. À la lumière de la thèse de l'" imprégnation éthique » cependant, la non reconnaissance constitutionnelle du Québec par le Canada apparaît comme répréhensible, non parce qu'elle nierait un droit collectif, mais parce qu'elle contrevient à une obligation juridique, celle, pour toute organisation politique, de prendre en considération les contextes culturels ou nationaux singuliers à l'intérieur desquels doivent être interprétés et utilisés les principes constitutionnels institués. La thèse de l'" imprégnation éthique ", si elle est vraie, montre finalement que le recours à des droits collectifs n'est pas indispensable pour la reconnaissance des minorités nationales, qu'elle peut se déduire directement de revendications juridiques, c'est-à-dire de l'obligation, pour tout législateur, de procéder à une contextualisation importante des normes du droit en regard des particularités, telles que l'existence de minorités nationales, que présentent les milieux de vie dans lesquels il doit légiférer.

(3) Le respect de l'intégrité des formes de vie collectives se déduit finalement d'une troisième thèse centrale que nous avons également eu l'occasion d'examiner, que nous pouvons appeler démocratique ou " républicaine ». Elle affirme l'interpénétration de l'autonomie privée et de l'autonomie publique, interpénétration créant un lien interne nécessaire entre État de droit et démocratie que méconnaîtrait, selon Habermas, la doctrine libérale traditionnelle. Le système des droits individuels ne peut être réalisé de manière conséquente que si les destinataires des droits subjectifs jouissent, non seulement d'une autonomie privée, mais également d'une autonomie publique, que s'ils peuvent se concevoir comme les auteurs des droits dont ils sont porteurs à titre de sujets de droit privés. Et cette autonomie publique ne peut à son tour être activée que grâce à leur participation démocratique, à des débats publics à propos de l'interprétation de leurs besoins et des conditions significatives de l'exercice de leurs droits.

Les citoyens du Québec ont clairement fait savoir quels étaient leurs besoins : vivre publiquement en français et pouvoir bénéficier d'une plus grande autonomie politique et administrative que les autres provinces afin de 
garantir leur culture publique commune. Ces revendications forment, depuis plus d'une trentaine d'années, les conditions minimales incontournables d'un exercice sensé de leurs droits, les conditions sans lesquelles ils ne pourraient se concevoir comme les véritables auteurs des lois auxquelles ils se soumettent. Elles ont fait l'objet de trois consultations publiques : les deux référendums de 1980 et 1995, tenus au Québec, ainsi que le référendum pancanadien de Charlottetown en 1993. Malheureusement, ces consultations n'ont pas permis de déterminer dans quel cadre les Québécois désirent exercer leur autonomie : est-ce sous forme d'État «particulier » au sein de l'État canadien ou d'État indépendant ? Tant et aussi longtemps qu'ils ne se seront pas mis au clair sur ces questions, les Québécois ne pourront vraisemblablement pas jouir pleinement de l'autonomie politique à laquelle ils aspirent et se sentiront aliénés dans le contexte canadien. À la lumière de la thèse républicaine cependant, et quelles que soient les décisions que prendront les Québécois dans l'avenir, leurs revendications ne requièrent, pour être prises en considération et reconnues, d'autre forme de justification que la procédure démocratique elle-même, que les délibérations qu'ils mènent et mèneront dans l'espace public, dans les arènes régionales, nationales et internationales, là où s'exerce, non pas leur autonomie « nationale » en tant que peuple, mais leur autonomie politique, leur "liberté républicaine " à titre de citoyens. C'est donc, exactement comme ils l'ont fait jusqu'à maintenant au moyen de consultations populaires et de référendums, par l'activation de leurs libertés politiques, par des débats publics, en particulier par des discussions éthicopolitiques portant sur ce qu'ils sont et veulent être et sur l'interprétation de leurs besoins, que les Québécois parviendront à clarifier entre eux le sens de leurs aspirations et à convaincre le reste des Canadiens du devoir de les reconnaître. Nul besoin ici de recourir à des «droits collectifs » à l'autodétermination nationale : seul l'exercice plein et entier des libertés démocratiques dont disposent déjà les Québécois à titre de citoyens suffit. Si elle est vraie, la thèse habermassienne du lien conceptuel unissant autonomie privée et publique, État de droit et démocratie montre finalement que ce qui manque à la notion de droits individuels pour que soient assurées les conditions de leur réalisation conséquente et intégrale, ce n'est pas un concept (substantiel) de droit collectif à l'autodétermination nationale, mais plutôt un concept (délibératif ou procédural) de participation démocratique ${ }^{27}$.

\subsection{Les avantages de la position de Habermas.}

Comme le démontrent suffisamment les trois thèses centrales de la théorie du droit et de la démocratie de Habermas que nous venons d'examiner, et comme le révèlent également les implications que nous en avons tiré à chaque

27. Sur cette distinction, voir plus haut la différence tracée par Habermas entre " autonomie nationale » et « autonomie républicaine », section 1.1. 
fois à propos de la situation qui prévaut actuellement au Québec et au Canada, la théorie individualiste des droits n'est en aucun temps incompatible avec la prise en considération des identités culturelles ou nationales ou avec le respect de l'intégrité des formes de vie collectives. Ceux-ci découlent au contraire d'une réalisation conséquente du système des droits fondamentaux lui-même. La position de Habermas nous semble des plus solides, conceptuellement parlant, et nous la jugeons supérieure à celle de Taylor, qui est pour sa part favorable à l'existence de droits collectifs de portée équivalente aux droits individuels. Trois raisons principales nous incitent à endosser la position de Habermas :

1) L'idée même de droits collectifs est problématique et sujette à caution $^{28}$. Bien que plusieurs projets de déclaration de droits collectifs soient présentement à l'étude aux Nations Unies (notamment à propos des droits aborigènes et des droits linguistiques), aucun n'a encore été officiellement adopté ou fait consensus ${ }^{29}$. Le seul droit collectif internationalement reconnu (en premier lieu par la Charte des droits de l'ONU) est le droit à l'autodétermination des peuples. Seulement, ce droit a été pensé en fonction des mouvements de décolonisation qui se sont multipliés après la seconde guerre mondiale. Il est peu probable qu'il puisse servir d'assise à la justification d'une plus large autonomie, voire de l'indépendance politique complète du Québec, à moins qu'il soit possible de démontrer, ce qui serait étonnant, que le Canada exerce sur lui une forme de domination de type colonial ${ }^{30}$. Mais ce n'est pas en premier lieu ce type de droit que des penseurs comme Charles Taylor ont en vue : ce qu'ils ont en vue, ce sont des droits humains collectifs de type culturel, des droits qui garantiraient en quelque sorte la survie à long terme de certaines communautés sociales et politiques minoritaires, comme le Québec, dont l'existence culturelle est menacée. À la différence du droit à l'autodétermination cependant, le droit à la survie culturelle des peuples n'est inscrit nulle part, dans aucune charte internationale des droits, et sa justification est rendue d'autant plus difficile que ce type de droit s'accorde

28. Michel Seymour, bien qu'il soit un farouche partisan des droits collectifs, semble néanmoins reconnaitre ce point. Voir Seymour, La nation en question, p. 35 et p.163.

29. Par exemple, la Déclaration universelle des droits linguistiques des peuples acclamée à Barcelone en 1996, approuvée par l'UNESCO et présentement à l'étude à l'ONU, considère ces droits comme étant tout à la fois "collectifs" et "individuels ". Pour une analyse intéressante des difficultés posées par les tentatives récentes d'intégration des droits collectifs au droit international, voir Anaya, S. J., "The Capacity of International Law to Advance Ethnic or Nationality Claims » dans Kymlicka, The Rights of Minority Cultures, p. 321-330.

30. Le principe de l'autodétermination des peuples ne peut donc justifier à lui seul la sécession d'une entité politique et la multiplication des mouvements sécessionnistes à travers le monde a amené certains auteurs, comme Allen Buchanan, à élaborer des principes normatifs, applicables en droit international, visant à les encadrer juridiquement de manière plus claire. Voir en particulier son étude "Self-Determination, Secession and the Rule of Law » dans McKim, R. et McMahan, J., dir., The Morality of Nationalism, New York, Oxford University Press, 1997, p. 301-323, en particulier p. 305-312 
très mal avec le système des droits actuellement reconnu dont la structure est individualiste. C'est pourquoi il constitue un appui bien fragile aux revendications autonomistes du Québec.

2) Naturellement, le fait que des droits collectifs d'une portée analogue aux droits individuels en matière de langue et de culture ne soient pas présentement pleinement institués au plan international ne constitue pas un argument suffisant pour les discréditer : il est parfaitement possible, à court ou à moyen terme, que des résolutions en leur faveur soient adoptées. Dans cet esprit, le Québec n'aurait-t-il pas raison, faisant preuve d'avant-gardisme et à défaut de bénéficier lui-même de tels droits collectifs de la part du Canada, d'en faire bénéficier à la communauté anglophone et aux onze nations autochtones présentes sur son territoire, donnant ainsi l'exemple de la marche à suivre ? Selon nous, agir de cette façon serait une erreur et nous nous accordons tout à fait ici avec les propos de Claude Bariteau : «[...] les membres des minorités ethniques et des communautés culturelles s'estimeront toujours légitimés de demander une reconnaissance similaire à celle que reçoivent les nations autochtones et la minorité anglophone ${ }^{31}$. » Il risque, en d'autres termes, d'y avoir des débordements et de la surenchère en faveur des droits collectifs de la part des autres groupes culturels présents sur le territoire québécois. Mais - l'objection est prévisible - une politique culturelle fondée exclusivement sur les droits individuels ne risque-t-elle pas, de son côté, de conduire au «melting pot » culturel ? Ne risque-t-elle pas de reproduire au Québec même la politique du multiculturalisme du Canada et d'être aveugle aux particularités historiques que présentent la minorité anglophone et les nations autochtones par rapport aux autres groupes ethniques et culturels issus de l'immigration ? Cela est parfaitement possible. On ne peut savoir a priori quelle sera la configuration des groupes culturels en présence sur le territoire québécois à moyen ou à long terme. Mentionnons cependant que, comme nous l'avons suffisamment montré, le système des droits individuels n'est pas incompatible avec la prise en compte des particularités historiques que présentent certaines communautés culturelles ou nationales minoritaires. De ce point de vue, ce que nous avons dit des relations entre le Canada et le Québec vaut également, de façon pour ainsi dire symétrique, pour les relations entre ce dernier et ses propres minorités nationales. C'est, conformément à l'esprit des thèses de Habermas que nous avons examinées et avec lesquelles nous nous accordons, non par l'attribution paternaliste d'un système de droits collectifs risquant de créer des enclaves, mais par la valorisation de la participation des minorités nationales au processus démocratique, participation leur permettant à tout moment de faire valoir leurs besoins et leurs aspirations propres, qu'elles pourront au mieux s'intégrer aux institutions publiques communes tout en préservant leur différence, que ce soit par rapport à la culture nationale majoritaire ou

31. Voir Bariteau, «Pour une conception civique du Québec » p. 142. 
par rapport aux autres groupes culturels minoritaires. L'État québécois peut certes, dans cet esprit, consentir à certaines mesures juridiques particulières réclamées par les Anglo-Québécois ou les nations autochtones en vue de favoriser leur autonomie (compromis plus souples en matière de législation linguistique, décentralisation des pouvoirs, autogestion gouvernementale, etc.), mais ces mesures doivent être d'une portée restreinte et rester subordonnées aux droits individuels ${ }^{32}$. L'État québécois peut également reconnaître leur existence collective dans une constitution ${ }^{33}$, mais il ne peut traduire cette reconnaissance dans une garantie juridique à la survie culturelle, c'est-à-dire dans l'enchâssement constitutionnel d'une série de droits collectifs ayant prépondérance sur les droits civiques individuels et destinés à protéger l'intégrité de leur tradition linguistique ou culturelle particulière au détriment de telle autre. Une telle politique discriminatoire risque de ghettoïser les minorités nationales et de générer des tensions entre elles et les autres groupes culturels.

3) Ceci nous mène tout droit à notre dernier point. Même si le Québec arrivait à ses fins en évitant les conflits et enchâssait - comme c'est son intention - dans la Constitution d'un Québec souverain les droits collectifs des Anglo-Québécois et des onze nations autochtones, un dernier argument, apporté par Habermas lui-même et qui nous apparaît le plus important dans notre contexte, nous incite à la plus grande prudence : « [...] la protection des formes de vie et des traditions formatrices de l'identité est, en dernière instance, au service de la reconnaissance de ses membres; il ne s'agit pas d'une protection administrative des espèces menacées. Le point de vue écologique de la conservation des espèces n'est pas transposable aux cultures $^{34}$. " En d'autres termes, à la différence des espèces animales, les cultures doivent pour se reproduire compter sur l'autonomie et la volonté de leurs membres, ceux-ci doivent être convaincus de la valeur de leurs traditions, de la nécessité de les poursuivre et ce n'est que dans ces conditions qu'ils peuvent avoir la motivation nécessaire pour les développer. Dans ce contexte, il ne fait tout simplement pas sens de chercher à garantir artificiellement, au moyen du droit, la préservation d'une culture : ceci irait à l'encontre de toute forme d'appropriation critique des héritages culturels, de toute possibilité de transformation, d'évolution, de progrès, non seulement des mentalités et des croyances mais de la langue maternelle elle-même, qui est une condition incontournable à la vitalité même d'une culture. Les cultures ne sont pas des biens fixes qu'il nous faut préserver de toute altération à la manière d'oeuvres d'art dans un musée. Une protection administrative

32. Sur le statut et la portée de ces mesures, voir note 24.

33. Attention ici : cette reconnaissance n'implique que l'existence de groupes culturels déterminés sur un territoire, non des droits collectifs correspondants.

34. "Kampf um Anerkennung », p. 259 (trad. franç. p. 226). Pour l'ensemble de l'argument, voir p. 257-264 (trad. franç. p. 224-231). 


\section{$396 \cdot$ Philosophiques / Automne 2000}

de la culture serait en fin de compte incompatible avec l'exigence démocratique elle-même, c'est-à-dire avec la liberté et l'autonomie sans lesquelles les membres d'une culture ne pourraient se concevoir comme ses auteurs et ne pourraient participer volontairement à son édification, à sa transformation ou, s'il le faut, à l'abandon de certaines de ses composantes. Elle risque de conduire à cela même que cherchent à éviter les promoteurs des droits collectifs culturels : à une forme de folklorisation des langues et des cultures.

\section{Vers une réhabilitation de l'État-nation}

Nous avons insisté dans la section précédente sur ce qui nous apparaît constituer les éléments forts, avec lesquels nous sommes en bonne partie d'accord, de la conception que se fait Habermas des revendications nationalistes à la lumière de sa théorie du droit et de la démocratie. Ceux-ci consistent à montrer qu'il n'est ni utile ni nécessaire de recourir à des droits collectifs pour assurer la reconnaissance des formes de vie culturelles ou nationales minoritaires à partir du moment : (1) où l'on modifie le modèle libéral de la citoyenneté en faveur d'un modèle où les liens entre l'État de droit et l'autonomie politique, la participation démocratique des citoyens sont pris en considération ; (2) où l'on élargit la lecture libérale du système des droits en faveur d'une lecture non restreinte, où les droits individuels ne sont plus déconnectés de leur contexte d'application, c'est-à-dire des contextes culturels ou nationaux particuliers auxquels le système juridique doit rester sensible. À partir du moment où les points (1) et (2) sont pris au sérieux, bon nombre des problèmes vécus par les minorités nationales peuvent se résoudre de manière pacifique à l'intérieur même des systèmes fédératifs, sans qu'il soit nécessaire pour cela de recourir à la sécession, puisque des formes suffisantes d'autonomie, administrative ou culturelle, leur sont accordées. Habermas rejoint là à sa manière, ni plus ni moins, les analyses de libéraux «souples » comme Will Kymlicka ou Allen Buchanan. À partir du moment où les points (1) et (2) sont pris au sérieux, on peut projeter, pour le Québec, l'idéal d'une fédération ou d'une confédération canadienne multinationale fortement décentralisée, voire asymétrique, qui serait fondée, comme toujours, sur une charte des droits individuels, mais qui devrait néanmoins demeurer ouverte et sensible, en raison de l' "imprégnation éthique » des systèmes juridiques, aux différences nationales et aux histoires nationales particulières, à la lumière desquelles seraient interprétés et appliqués les principes universalistes de cette charte, faisant de son côté l'objet d'un patriotisme constitutionnel. Mais à l'aide des points (1) et (2), on peut également projeter pour le Québec l'idéal d'un État-nation indépendant qui serait tout aussi fortement décentralisé, ouvert et intégrateur à l'endroit de ses propres minorités que pourrait l'être le Canada à son endroit. Pourquoi donc le refus de cette alternative par Habermas? 
Ce refus révèle une faiblesse importante de sa position : prétendre que les problèmes des minorités nationales ont tout avantage à se régler dans un cadre fédéral et que leur intégration à des systèmes fédératifs constitue à toute fin pratique l'unique voie raisonnable de solution aux formes de domination ou de discrimination plus ou moins occultées dont elles sont victimes. Sa faiblesse est son refus de voir dans la constitution d'un nouvel État-nation une solution tout aussi acceptable que la voie fédérative. Certes, toutes les minorités ne peuvent, ni ne veulent se constituer en État-nation. Pour certaines minorités, le fédéralisme supranational ouvert et intégrateur que propose Habermas s'offre sans doute comme la meilleure option envisageable. Mais tel n'est manifestement pas le cas pour toutes les minorités. Quand l'Étatnation s'offre comme un horizon viable, comme au Québec, il n'est pas du tout évident, lorsqu'il est question de survie culturelle, lorsque l'identité collective d'un peuple est mise en péril, que la voie fédérative présentera partout et toujours la meilleure solution. L'erreur de Habermas (qui rejoint ici les positions de Buchanan) est de prétendre que oui. Contre cette vue des choses, nous allons dans les lignes qui suivent tenter d'expliquer très brièvement pourquoi l'État-nation nous semble devoir être considéré comme un idéal politique légitime, comme une voie de solution envisageable aux problèmes des minorités nationales (3.1.); nous tirerons par la suite les conséquences de nos analyses pour ce qui est de la place et du rôle de l'État-nation dans le contexte de la mondialisation (3.2.).

\section{1.}

Comme l'a montré Habermas lui-même dans d'autres contextes, en particulier dans ses travaux sur l'évolution sociale et le développement des identités collectives $^{35}$, la forme moderne de l'État-nation possède cette particularité de rendre possible la constitution d'une double identité : une identité conventionnelle, ou « identité par rapport à des rôles », fondée sur l'appartenance à une communauté culturelle, linguistique, nationale concrète (le stade 4 dans l'échelle de Kohlberg); et une identité postconventionnelle, ou " identité du moi », fondée sur des principes de justice universalistes, c'està-dire sur les droits de l'homme et les principes constitutifs de l'État de droit démocratique (le stade 5 dans l'échelle de Kohlberg). Les membres de l'Étatnation moderne sont ainsi membres d'une communauté politique présentant un double visage : d'une part, celui d'une communauté « nationale » ayant une tradition ainsi qu'une couleur culturelle ou linguistique particulières ; mais d'autre part, et tout à la fois, celui d'une communauté "démocratique », lieu de formation d'une identité politique abstraite, qui accorde à

35. Voir, par exemple, son étude "Einleitung: Historischer Materialismus und die Entwicklung normativer Strukturen", dans Habermas, J., Zur Rekonstruktion des Historischen Materialismus, p. 28-29 (trad. franç. par J.-R. Ladmiral et M.B. de Launay dans Habermas, J., Après Marx, Paris, Fayard, 1985, p. 57-59). 
tous sans exception le statut de citoyen jouissant à la fois d'une autonomie privée et d'une autonomie publique.

C'est sur cette double identité rendue possible par l'État-nation que se fonde la thèse de Habermas sur les rapports entre nation et démocratie, thèse en partie vraie et en partie inexacte. Il est vrai que la "fusion » que réalise historiquement l'État-nation entre l'identité nationale et la citoyenneté démocratique est purement contingente. Conceptuellement, il n'existe en effet aucune relation de nécessité entre les deux : la «nation sans démocratie » et la "démocratie sans nation »sont deux possibilités parfaitement envisageables. On remarquera cependant que seul le premier cas présente des occurrences historiques (le nationalisme ethnique). Une démocratie purement cosmopolite reste encore à naître. Cela ne doit pas surprendre : si l'on s'en tient au schéma de Kohlberg, l'identité nationale et l'identité fondée sur une citoyenneté cosmopolite renvoient à des étapes différentes dans la constitution des identités individuelles et collectives. Dans cette perspective, s'il est juste de soutenir que la fusion que réalise l'État-nation entre identité nationale et citoyenneté démocratique est, d'un point de vue strictement conceptuel, contingente, il en va autrement d'un point de vue diachronique. Sur le chemin allant de la pure nation à la pure démocratie, l'État-nation joue en effet un rôle et occupe une position, sinon absolument nécessaires, à tout le moins décisifs. Il forme sans doute, tant au plan historique que biographique, le cadre politique le plus favorable à la transition d'une identité nationale à une identité politique cosmopolite. Sans les principes universalistes de l'État de droit, la voie démocratique aurait été barrée pour les nations modernes, qui se seraient alors repliées sur une identité purement ethnique avec pour seul horizon celui d'une politique de la puissance à l'endroit des autres nations. Mais d'autre part, une démocratie purement cosmopolite, sans forme préalable d'organisation politique protectrice des identités nationales, risquerait d'abandonner ces identités à leur sort, n'offrant d'elle-même aucun cadre politique défini permettant leur protection et leur reconnaissance. L'État-nation semble donc se présenter comme un point de jonction, comme une forme politique de transition des plus importantes, celle permettant sans doute le mieux, à la fois de surmonter les particularismes nationaux en accordant à tous le statut de citoyen susceptible de s'autodéterminer dans les sphères privées et publiques, mais également de compenser l'abstraction engendrée par l'universalisme d'une citoyenneté purement cosmopolite, en fournissant cette fois un cadre politique déterminé pour la protection des identités culturelles et nationales. C'est parce qu'il remplit cette double fonction, qu'il est à la fois lieu de constitution d'une première forme d'autolégislation démocratique à grande échelle et lieu de protection des traditions nationales, que l'État-nation est sans doute devenu ce qu'il est aujourd'hui : une norme internationale, une entité juridique et une réalité politique reconnues partout à travers le monde, représentant ainsi une force d'attraction pour tous les mouvements nationalistes. 
On peut conclure de ces réflexions sommaires que c'est le modèle purement ethnique de l'État-nation que Habermas a en vue lorsqu'il rejette le nationalisme, alors que ses travaux eux-mêmes nous conduisent à une compréhension beaucoup plus nuancée de cette forme d'organisation politique. Le modèle présenté ici peut tout à fait bien s'acquitter des tâches que Habermas confie actuellement seulement aux États fédérés et il possède la plupart de ses vertus. L'idéal républicain d'une forme d'organisation politique fondée sur une charte des droits individuels, faisant l'objet d'un patriotisme constitutionnel mais demeurant néanmoins ouverte et sensible, en raison de l'« imprégnation éthique » des systèmes juridiques, aux différences culturelles et aux histoires nationales particulières, peut être le fait, non seulement d'une fédération multinationale fortement décentralisée (comme pourrait l'être le Canada), mais également d'un État-nation indépendant (comme pourrait l'être le Québec), tout aussi fortement décentralisé, ouvert et intégrateur à l'endroit de ses propres minorités natives (en l'occurrence ici, les onze nations autochtones). Cette possibilité n'est plus à exclure à partir du moment où les composantes civiques ou républicaines et les composantes ethno-nationales d'un État, l'intégration politique et l'intégration culturelle des citoyens sont clairement détachées et tenues à distance les unes des autres, comme le recommande Habermas. Le modèle de l'État-nation décrit plus haut remplit tout à fait cette exigence en rendant possible, voire obligatoire, la cohabitation pacifique d'une nation majoritaire avec plusieurs autres nations ou cultures minoritaires à l'intérieur d'un même territoire. Le modèle républicain et non purement ethnique de l'État-nation n'est donc pas incompatible avec le multiculturalisme, et la possibilité de sa transformation en véritable État multinational reste toujours ouverte.

\section{2 .}

Une fois réhabilité l'État-nation dans son statut et dans son rôle - et sous réserve de précisions supplémentaires - il convient maintenant de revenir sur le problème de la mondialisation. La perte de contrôle de l'État-nation sur les effets globaux induits par la dénationalisation de l'économie fait à peu près partout, aujourd'hui, consensus : les moyens politiques offerts par l'Étatnation doivent être dépassés. Mais cela ne signifie pas que la structure de l'État-nation doit être abandonnée, comme semble le suggérer Habermas. Il importe donc de concevoir ses relations avec des institutions supranationales, ainsi que sa place dans le contexte d'une démocratie cosmopolitique, d'une manière différente de celle préconisée par Habermas. Nous ne donnerons ici que quelques pistes.

Plutôt que de concevoir l'avenir de l'État-nation selon le modèle de sa dissolution progressive dans des unités fédératives supranationales toujours plus vastes, il nous semble plus raisonnable et plus réaliste de le concevoir selon le modèle d'une souveraineté partagée. Ce modèle a l'avantage de 
répondre adéquatement aux aspirations, non seulement de bon nombre d'États souverains actuels, qui sont sans doute disposés à un certain nombre de transferts de pouvoir mais ne désirent aucunement renoncer entièrement à leur souveraineté, mais également de la plupart des mouvements nationalistes, pour lesquels la constitution d'un État-nation n'est pas une fin en soi, mais un tremplin, une étape en vue d'une participation à des unités supranationales plus englobantes (c'est le cas du nationalisme québécois ${ }^{36}$ ). Le modèle d'intégration européenne proposé dans certains textes par Habermas ${ }^{37}$ semble, de son côté, laisser peu d'initiative à l'État-nation. Alors que plusieurs observateurs se réjouissent du fait que le traité de Maastricht ne vienne pas, du moins en principe, limiter les marges de manoeuvre de l'État-nation au plan politique, par exemple en ce qui a trait aux questions de justice, de politique intérieure et extérieure ou à celles liées à l'entretien de forces armées, et qu'il respecte donc la souveraineté politique des États membres, Habermas va pour sa part à contre-courant : les États-nations européens doivent consentir à un transfert important de souveraineté s'ils veulent qu'un futur Parlement européen soit doté de véritables pouvoirs politiques et qu'un processus démocratique puisse avoir quelque efficace à l'échelle européenne. Il juge donc que les restrictions mentales contre un tel transfert ne sont pas fondées et qu'elles ne pourront selon lui être levées que si les membres des États-nations d'Europe acceptent de transiger leur identité politique nationale pour une identité politique postnationale, et si la conception trop concrétiste de la souveraineté populaire sur laquelle ils s'appuient tacitement est abandonnée en faveur d'une conception plus abstraite, qui est la conception procédurale que défend Habermas lui-même. Le problème ici est de savoir jusqu'où, au juste, vont les transferts de souveraineté réclamés par Habermas. Il n'est pas clair sur ces questions. Si un transfert de souveraineté en matière militaire (défense, alliances, maintien de la paix, etc.) ou en matière économique (politique monétaire) nous semble à première vue poser peu de problème, il n'en va pas de même pour les transferts en matière d'éducation, de santé, de culture, d'immigration, etc. Ces domaines pourraient-ils être les cibles d'une future "politique européenne », des domaines de législation clef d'un futur parlement européen où siégeraient de futurs partis européens ? Son attitude négative à l'endroit de l'État-nation n'annonce rien, ici, de rassurant.

Pour ce qui est, finalement, de la place de l'État-nation à l'intérieur d'une éventuelle démocratie cosmopolitique, notre lecture diffère quelque

36. La plupart des intellectuels nationalistes québécois s'entendent aujourd'hui sur la nécessité de formes de partenariats et de partages de souveraineté à différents paliers de gouvernements. Sur cette question, voir Bourque, G. et Duchastel, J., L'identité fragmentée, Saint-Laurent, FIDES, 1996.

37. Voir par exemple "Braucht Europa eine Verfassung ? Eine Bemerkung zu Dieter Grimm », dans EdA, p. 185-191 (trad. franç. dans L'intégration républicaine, p. 151-157). 
peu de celle qu'en donne Habermas. Celui-ci suggère de considérer une future union cosmopolitique, en opposition à Kant, non selon le modèle d'une fédération d'États, mais d'une fédération de citoyens du monde (Weltbürgern) qui éliraient leurs représentants, non via leurs États respectifs, mais directement dans un parlement mondial ${ }^{38}$. L'intention de Habermas ici est claire : remplacer les États-nations, sujets collectifs du droit international, par les sujets de droit individuels et ménager pour ceux-ci une appartenance directe à une association cosmopolitique. Si nous sommes d'accord avec ce modèle, l'interprétation que nous en faisons diffère de celle de Habermas. Celui-ci voit en effet l'État-nation comme un obstacle, à surmonter, dans la constitution d'une telle association : la fédération des citoyens cosmopolites doit garantir que les droits individuels auront primauté sur les droits collectifs à l'autodétermination nationale. Mais ce faisant, Habermas limite le rôle de l'État-nation à celui d'un simple instrument de l'affirmation nationale. Il semble ne pas voir qu'il est aussi le lieu de l'autolégislation démocratique des citoyens. Envisagé sous cet angle, l'État-nation n'est pas opposé ou incompatible avec une association de citoyens cosmopolites : celle-ci se présente au contraire comme une étape supplémentaire dans le processus d'autolégislation des citoyens, étape qui, en franchissant les frontières de l'État-nation, ne fait que compléter et étendre à d'autres domaines la pratique d'autodétermination démocratique déjà à l'œuvre à l'intérieur des frontières nationales. Loin d'être incompatible avec une association cosmopolite, l'État-nation se présente au contraire, conformément à nos thèses antérieures, comme l'une de ses conditions de possibilité, comme une étape charnière dans le processus d'apprentissage, par les citoyens, de l'exercice de leur autonomie politique.

38. Voir "Kants Idee des ewigen Friedens", dans EdA, p. 210 sq. (trad. franç. dans L'intégration républicaine, p. 179 sq.) ; "Die postnationale Konstellation ", dans DpK, p. 160 $s q$. 